\title{
Renormalization-group approach to two-dimensional quantum models
}

\author{
J. Rogiers*† \\ Physics Department, Massachusetts Institute of Technology, Cambridge, Massachusetts 02139 \\ R. Dekeyser ${ }^{\dagger}$ \\ Physics Department, University of Wisconsin, Madison, Wisconsin 53706 \\ (Received 10 June 1975; revised manuscript received 14 October 1975)
}

\begin{abstract}
The two-dimensional $X Y$ and Heisenberg models are studied with a quantum-mechanical generalization of the Niemeijer and Van Leeuween renormalization-group method. In second-order cumulant expansion a fixed point exists for the $X Y$ model, giving a critical temperature at $J / k T_{c} \cong 0.87 \pm 0.05$. For the Heisenberg model we find a fixed point at $K^{*}=2.8$. This result may not be reliable, since the truncation of the cumulant expansion is only valid for small $K$.
\end{abstract}

\section{INTRODUCTION}

Although Mermin and Wagner ${ }^{1}$ showed that no long-range order can exist in a variety of twodimensional models, including the $X Y$ and Heisenberg models, there is strong evidence, mainly from series expansions, ${ }^{2,3}$ for the existence of pseudo-Curie points in these models. For the $X Y$ model on the triangular lattice, Betts et al. ${ }^{4}$ give $K_{0} \simeq 0.667 \pm 0.003$ and $\gamma \simeq 1.50 \pm 0.02$ as their best estimates. Series analysis for the two-dimensional Heisenberg model did not give a convincing argument for the presence of a singularity. ${ }^{3}$

It is possible to study these models with a quantum-mechanical generalization of the Niemeijer and Van Leeuwen ${ }^{5}$ approach to the renormalization-group calculations. ${ }^{6}$ In this method, we construct a transformation from the original basis vectors in spin space $\left|\left\{s_{j}\right\}\right\rangle$ (where $s_{j}= \pm \frac{1}{2}$ indicates the $z$ component of the spin on the $j$ th lattice site) to new basis vectors characterized by a cell spin variable $s_{J}$, and some dummy variable $\tau_{J}$ (for the $J$ th cell). Since such a transformation is not unique, let us indicate the possible new basis vectors by $\left|\left\{s_{J}, \tau_{J}\right\} ; k\right\rangle$, where $k$ runs over all possible transformations. The partition function $Z^{-}$ may then be written as

$$
\begin{aligned}
Z & =\operatorname{Tr} e^{\Re C} \\
& =\sum_{\left\{s_{j}\right\}}\left\langle\left\{s_{j}\right\}\left|e^{\mathfrak{K C}}\right|\left\{s_{j}\right\}\right\rangle \\
& =\sum_{k} p_{k} \sum_{\left\{s_{J}, \tau_{J}\right\}}\left\langle\left\{s_{J}, \tau_{J}\right\} ; k\left|e^{\mathfrak{K}}\right|\left\{s_{J}, \tau_{J}\right\} ; k\right\rangle .
\end{aligned}
$$

The $p_{k}$ may be chosen arbitrarily apart from the constraint

$$
\sum_{k} p_{k}=1
$$

They should, however, be such that the symmetries of the Hamiltonian are preserved in the transformation. We have

$$
Z=\sum_{\left\{s_{J}\right\}}\left\langle\left\{s_{J}\right\}\left|e^{\Im e^{\prime}}\right|\left\{s_{J}\right\}\right\rangle,
$$

where

$$
\begin{gathered}
\left\langle\left\{s_{J}\right\}\left|e^{\Im C}\right|\left\{s_{J}^{\prime}\right\}\right\rangle= \\
=\sum_{k} p_{k} \sum_{\left\{\tau_{J}\right\}}\left\langle\left\{s_{J}, \tau_{J}\right\} ; k\left|e^{\Im C}\right|\left\{s_{J}^{\prime}, \tau_{J}\right\} ; k\right\rangle \\
\equiv\left\langle\left\{s_{J}\right\}\left|\operatorname{Tr}^{\prime} e^{\Im C}\right|\left\{s_{J}^{\prime}\right\}\right\rangle . \\
\text { II. } X Y \text { MODEL }
\end{gathered}
$$

We have studied the $X Y$ model with Hamiltonian

$$
\mathfrak{H}^{X Y}=K \sum_{\langle i j\rangle}\left(S_{i}^{+} S_{j}^{-}+S_{j}^{+} S_{i}^{-}\right)
$$

where the sum runs over all nearest-neighbor pairs (only once) on a triangular lattice. We have chosen the cells as in Ref. 5, and may write

$$
\mathfrak{H}^{X Y}=\mathfrak{H}_{0}^{X Y}+V,
$$

where $\mathcal{F}_{0}^{X Y}$ contains all intracell interactions.

We have taken several sets of renormalization transformations, obtaining slightly varying results. We mention two of them: (i) the spin inversion symmetric set

$$
\begin{aligned}
& |+; 1\rangle=|++-\rangle, \quad|+; 2\rangle=|+-+\rangle, \quad|+; 3\rangle=|-++\rangle, \quad|+; 4\rangle=|+++\rangle, \\
& |-; 1\rangle=|--+\rangle, \quad|-; 2\rangle=|-+-\rangle, \quad|-; 3\rangle=|+--\rangle, \quad|-; 4\rangle=|---\rangle
\end{aligned}
$$

and (ii) a combination of the following two sets with equal weights $p_{k}=\frac{1}{2}$, 


$$
\begin{aligned}
k=1: & |+; 1\rangle=|++-\rangle, \quad|+; 2\rangle=|+-+\rangle, \quad|+; 3\rangle=|-++\rangle, \quad|+; 4\rangle=|+++\rangle, \\
& |-; 1\rangle=|-+-\rangle, \quad|-; 2\rangle=|+--\rangle, \quad|-; 3\rangle=|--+\rangle, \quad|-; 4\rangle=|---\rangle ; \\
k=2: & |+; 1\rangle=|++-\rangle, \quad|+; 2\rangle=|+-+\rangle, \quad|+; 3\rangle=|-++\rangle, \quad|+; 4\rangle=|+++\rangle, \\
& |-; 1\rangle=|+--\rangle, \quad|-; 2\rangle=|--+\rangle, \quad|-; 3\rangle=|-+-\rangle, \quad|-; 4\rangle=|---\rangle .
\end{aligned}
$$

Each state $\left|\alpha_{1} \alpha_{2} \alpha_{3}\right\rangle$ is a direct product of eigenstates of the three $S^{\boldsymbol{z}}$ operators in a cell.

To obtain $\left(\mathcal{H}^{X Y}\right)^{\prime}$, we first write

$$
e^{{ }^{X X Y}}=e^{\mathfrak{F}_{0}^{X Y}} e^{W},
$$

where

$$
W=V+\frac{1}{2}\left[V, \mathcal{H}_{0}^{X Y}\right]-\frac{1}{12}\left[2 \mathcal{H}_{0}^{X Y}+V,\left[V, \mathcal{H}_{0}^{X Y}\right]\right]+\cdots,
$$

which follows from the Baker-CampbellHausdorff formula. ${ }^{7}$ Then

$$
\begin{aligned}
\left(\mathcal{F C}^{X Y}\right)^{\prime} & =\ln \left(\operatorname{Tr}^{\prime} e^{\mathcal{F}_{0}^{X Y}} e^{W}\right) \\
& =\ln \left(\operatorname{Tr}^{\prime} e^{\mathcal{F C}_{0}^{X Y}}\right)+\ln \left\langle e^{W}\right\rangle,
\end{aligned}
$$

where the cell-spin-operator-valued expectation value is defined as

$$
\langle A\rangle \equiv \operatorname{Tr}^{\prime} e^{\mathcal{F}_{0}^{X Y}} A\left(\operatorname{Tr}^{\prime} e^{{ }^{X} C_{0}^{X Y}}\right)^{-1} .
$$

This procedure is justified only if $\left(\operatorname{Tr}^{\prime} e^{\Im C} 0\right)$ is a multiple of the unit operator in cell spin space, which is indeed the case for this transformation. We apply a cumulant expansion to the last term in Eq. (9):

$$
\begin{aligned}
\ln \left\langle e^{W}\right\rangle= & \langle W\rangle+\frac{1}{2}\left(\left\langle W^{2}\right\rangle-\langle W\rangle^{2}\right) \\
& +\frac{1}{6}\left(\left\langle W^{3}\right\rangle-\frac{3}{2}\left\langle W^{2}\right\rangle\langle W\rangle\right. \\
& \left.\quad-\frac{3}{2}\langle W\rangle\left\langle W^{2}\right\rangle+2\langle W\rangle^{3}\right)+\cdots .
\end{aligned}
$$

All expectation values can be determined exactly, and we have calculated the renormalized interactions up to second order in $K .^{8}$ This induces nextand third-nearest-neighbor $X Y$ coupling, which we have given interaction coefficients $L$ and $M$, respectively, and also a $\left(S_{i}^{z} S_{j}^{z}\right)$ coupling for nearest neighbors with a coefficient $R$. The renormalized Hamiltonian is

$$
\begin{aligned}
\left(\mathcal{F C}^{X Y}\right)^{\prime}=\frac{1}{3} N[ & -\ln (h+g-f)+3 g_{1} R \\
+ & \left.\frac{3}{2} K^{2}\left(1-2 f^{4}+2 g\right)\right]+\mathcal{H}_{1}^{X Y},
\end{aligned}
$$

where $N$ is the number of lattice points. $\mathcal{F}_{1}^{X Y}$ is of the same form as $\mathcal{H}^{X Y}$ with interaction coefficients

$$
\begin{aligned}
& K^{\prime}=f^{2}(2 K+3 L+2 M)+2 f^{2}\left(1+2 g-2 f^{2}\right) K^{2}, \\
& L^{\prime}=f^{2} M+\frac{1}{2} f^{2}\left(1+14 g-8 f^{2}\right) K^{2}, \\
& M^{\prime}=2 f^{2}\left(2 g-f^{2}\right) K^{2}, \\
& R^{\prime}=2 h^{2} R+2\left(2 f^{4}-h^{2}\right) K^{2}, \\
& \text { re } \\
& g=q\left(e^{3 K}-1\right), \\
& h=q\left(e^{3 K}+3 e^{K}+2\right), \\
& q^{-1}=3\left(e^{3 K}+e^{K}+2\right), \\
& 4 g_{1}=h-2 f+2 g,
\end{aligned}
$$

Here

and for the transformations (6a) and (6b), respectively,

$$
\begin{aligned}
& f=2 q\left(e^{3 K}-1\right), \\
& f=q\left(2 e^{3 K}+1\right) .
\end{aligned}
$$

In each case, a nontrivial fixed point is found: at $K^{*}=0.8554, L^{*}=0.2131, M^{*}=0.0774$ and $R^{*}$ $=-0.2030(6 \mathrm{a})$, and at $K^{*}=0.7566, L^{*}=0.1502$, $M^{*}=0.0491$ and $R^{*}=-0.1362(6 \mathrm{~b})$.

Linearizing the equations around the fixed point gives us values for the eigenvalue related to the temperature $\lambda_{T}$, and an estimate of the critical temperature $\left(K_{C}\right){ }^{5}$ The magnetic eigenvalue is obtained by evaluating the linear part in $H$ of (12) if we include a term $H \sum_{j}\left(S_{j}^{+}+S_{j}^{-}\right)$in the Hamiltonian. We did this calculation up to second order in $K$. In Table I we give the results for $\lambda_{T}, \lambda_{H}, K_{C}$, and some exponents. The negative values for $\delta$ and $\eta$ might be due to the rather crude approximation. ${ }^{9}$ If these negative values should persist in better approximations, this would suggest that the phase transition is of a more complicated type. ${ }^{10}$

Other sets of transformations give results which are only slightly different from the previous values, and we may summarize them by

$$
\begin{aligned}
& K_{C}=0.87 \pm 0.05, \alpha=0.8 \pm 0.2 \\
& \gamma=2.0 \pm 0.1, \nu=0.6 \pm 0.1
\end{aligned}
$$

\section{HEISENBERG MODEL}

We have repeated the same procedure for the Heisenberg Hamiltonian. 


$$
\mathcal{H C}^{H}=2 K \sum_{\langle i, j\rangle}\left(S_{i}^{x} S_{j}^{x}+S_{i}^{y} S_{j}^{y}+S_{i}^{z} S_{j}^{z}\right)
$$

Using the transformations (6a) and (6b), we generate a renormalized interaction which is anisotropic in spin space, i.e., we obtain

$$
\left.(\mathcal{H C})^{H}\right)^{\prime}=\sum_{(i, j)}\left[2 K_{1}^{\prime}\left(S_{i}^{x} S_{j}^{x}+S_{i}^{y} S_{j}^{y}\right)+2 K_{2}^{\prime} S_{i}^{z} S_{j}^{z}\right]
$$

This anisotropy, introduced by the normalization, cannot be tolerated and must be removed. Its appearance is completely different from the appearance of next-nearest-neighbor interactions when starting from a nearest-neighbor Hamiltonian. ${ }^{5}$ Indeed, if there is a fixed point for the Heisenberg Hamiltonian, it should by symmetry reasons be an isotropic fixed point (or else there should be an infinity of them). It happens, however, that one can remove this artificially introduced anisotropy by using in the renormalization (3) more sets $\left|\left\{s_{J}, \tau_{J}\right\} ; k\right\rangle$ of new base vectors, chosen in a more symmetric way. This can be achieved by using either three transformation sets like (6a) (with equal weights $p_{k}=\frac{1}{3}$ ), or six sets like $(6 \mathrm{~b})$ (with $p_{k}=\frac{1}{6}$ ), which are obtained by interpreting the original base vectors in (6) as eigenstates of $S^{x}, S^{y}$, and $S^{\boldsymbol{z}}$, alternately.

This gives an isotropic interaction parameter $K^{\prime}$

$$
K^{\prime}=\frac{1}{3}\left(2 K_{1}^{\prime}+K_{2}^{\prime}\right)
$$

For these isotropic generalizations of the transformations (6a) and (6b), we obtain the renormalization-group equations (20) for the nearest-neighbor $(K)$, next-nearest-neighbor $(L)$, and thirdnearest-neighbor $(M)$ interaction parameters. $L$ and $M$ are supposed to be of order $K^{2}$.

TABLE I. Relevant eigenvalues, critical temperature, and critical exponents for the $X Y$ model for transformations (6a) and (6b).

\begin{tabular}{crr}
\hline \hline Transformation & $(6 \mathrm{a})$ & $(6 \mathrm{~b})$ \\
\hline$\lambda_{T}$ & 2.797 & 2.285 \\
$\lambda_{H}$ & 4.910 & 4.113 \\
$K_{C}$ & 0.917 & 0.816 \\
$\alpha$ & 0.932 & 0.671 \\
$\gamma$ & 2.026 & 2.093 \\
$\delta$ & -3.230 & -4.482 \\
$\eta$ & -1.794 & -1.149 \\
$\nu$ & 0.534 & 0.665 \\
\hline
\end{tabular}

$$
\begin{aligned}
K^{\prime}= & {\left[2 K+3 L+2 M+K^{2}(1+4 g)\right] f^{2} } \\
& -\frac{1}{3} K^{2}\left(6 f_{1}^{4}+4 f_{2}^{4}-4 f_{1}^{2} f_{2}^{2}\right), \\
L^{\prime}= & {\left[M+K^{2}\left(\frac{1}{2}+7 g\right)\right] f^{2}-\frac{4}{3} K^{2}\left(2 f_{1}^{4}+f_{2}^{4}\right), } \\
M^{\prime}= & 4 K^{2} f^{2} g-\frac{2}{3} K^{2}\left(2 f_{1}^{4}+f_{2}^{4}\right),
\end{aligned}
$$

with

$$
\begin{aligned}
& f^{2}=\frac{1}{3}\left(2 f_{1}^{2}+f_{2}^{2}\right), \\
& g=\frac{1}{6} \tanh \frac{3}{2} K, \\
& f_{2}=\frac{1}{2}+g
\end{aligned}
$$

and

$$
f_{1}=2 g \text {, }
$$

or

$$
f_{1}=\frac{1}{2} f_{2}
$$

for the generalizations of (6a) and (6b), respectively. With (24a) we find a nontrivial fixed point ${ }^{11}$ at $K^{*}=2.8170, L^{*}=0.5870$ and $M^{*}=-.00033$. With (24b), the nontrivial fixed point is located at $K^{*}=2.8147, L^{*}=0.5863$, and $M^{*}=-.00025$. The value of $K$ is rather large, so that the argument for the truncation of the cumulant expansion (11), namely, that higher-order interactions decay with a power of $K$, fails. Essentially, we make an expansion in $K$, a parameter which is not small at the fixed point; it may therefore be inappropriate to apply this method to the two-dimensional Heisenberg model.

\section{CONCLUSION}

We have generalized the renormalization-group approach of Niemeijer and Van Leeuwen to quantum-mechanical systems in two dimensions. For the $X Y$ model, we find a nontrivial fixed point, which suggests a possible phase transition. The values of the exponents are still questionable, and may give an indication of the strange nature of this phase transitions. For the Heisenberg model, the nontrivial fixed point is not consistent with the basic assumption justifying the truncation of the cumulant expansion, and the existence of a phase transition is questionable.

\section{ACKNOWLEDGMENTS}

The authors wish to thank Professor D. L. Huber and Professor H. E. Stanley for their hospitality at the University of Wisconsin and at MIT, and the Belgian Nationaal Fonds voor Wetenschappelijk Ondersock for financial help. 
*Research supported by the Belgian N. F. W. O.

$\dagger$ Permanent address: Institute for Theoretical Physics, Katholieke Universiteit Leuven, Celestijnenlaan $200 \mathrm{D}$, B-3030 Heverlee, Belgium.

${ }^{1}$ N. D. Mermin and H. Wagner, Phys. Rev. Lett. 17, 1133 (1966).

${ }^{2}$ H. E. Stanley and T. Kaplan, Phys. Rev. Lett. 17, 913 (1966).

${ }^{3}$ For a discussion of these analyses, see the review articles of D. D. Betts and G. S. Rushbrooke, G. A. Baker, Jr., and P. J. Wood, in Phase Transitions and Critical Phenomena; edited by C. Domb and M. S. Green (Academic, New York, 1974), Vol. 3.

${ }^{4}$ D. D. Betts, C. J. Elliott, and R. V. Ditzian, Can. J. Phys. 49, 1327 (1971).
${ }^{5}$ Th. Niemeijer and J. M.J. Van Leeuwen, Phys. Rev. Lett. 31, 1411 (1973); Physica (Utr.) 16, 425 (1974).

${ }^{6}$ For a review, see K. G. Wilson and J. Kogut, Phys. Rep. 12C, 75 (1974).

${ }^{7}$ See, e.g., R. M. Wilcox, J. Math. Phys. 8, 962 (1967). ${ }^{8}$ In first-order approximation, no fixed point could be found.

${ }^{9} \mathrm{~S} . \mathrm{Hsu}$, Th. Niemeijer, and J. D. Gunton, Phys. Rev. B 11,2699 (1975).

${ }^{10}$ J. M. Kosterlitz, J. Phys. C $\underline{7}, 1046$ (1974).

${ }^{11}$ Owing to a numerical error, the authors reported elsewhere [Proceedings of the IUPAP International Confer ence on Statistical Physics, Budapest, 1975 (unpublished), p. 86] the absence of a fixed point. 